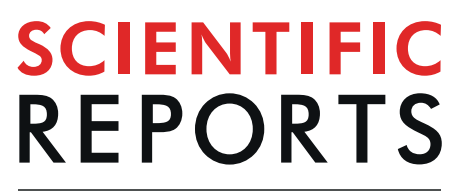

natureresearch

\title{
Controlling Nutritional Status (CONUT) score is a prognostic factor in patients with resected breast cancer
}

\begin{abstract}
Wen $\mathrm{Li}^{1}$, Min $\mathrm{Li}^{2}$, Ting Wang ${ }^{1}$, Guangzhi Ma ${ }^{1}$, Yunfu Deng ${ }^{1}$, Dan $\mathrm{Pu}^{1}$, Zhenkun Liu ${ }^{1}$, Qiang Wu ${ }^{1}$, Xuejuan Liu ${ }^{3}$ \& Qinghua Zhou ${ }^{1 *}$

The present study aimed to determine the correlation between controlling nutritional status (CONUT) and prognosis in resected breast cancer patients. Totally, 861 breast cancer patients with surgical resection in West China Hospital of Sichuan University between 2007 and 2010 were included. The relationship between CONUT and various clinicopathological factors as well as prognosis was evaluated. The results showed that the optimal cutoff value for CONUT to predict the 5-year survival was 3 and CONUT had a higher area under the ROC curve (AUC) for 5 -year disease free survival (DFS) and overall survival (OS) prediction compared with the neutrophil lymphocyte ratio (NLR) and prognostic nutritional index (PNI). High CONUT was significantly correlated with older age, lymph node involvement, advanced T-stage, and surgery type. In the multivariate analysis, CONUT-high patients had worse DFS and OS, when compared with CONUT-Iow patients. In conclusion, preoperative CONUT is a useful marker for predicting long term outcomes in breast cancer patients after curative resection.
\end{abstract}

Breast cancer is one of the most commonly diagnosed malignancies in women worldwide ${ }^{1}$. Although surgery is the main treatment for breast cancer, its clinical course remains unsatisfactory since an appreciable part of patients develop local recurrence or distal metastasis after resection ${ }^{2}$. Hence, it is vital to find out potential biomarkers to accurately predict the prognosis and provide comprehensive information for selecting appropriate treatment strategies.

It has been identified that the cancer prognosis is, to some extent, related to host status, including nutrition or inflammation ${ }^{3}$. Besides, poor nutritional condition may be correlated with the metabolic elevation and the immune-compromised status in cancer patients ${ }^{4,5}$. Previous studies have reported that preoperative nutritional status, including albumin, is related with the prognosis in several malignancies ${ }^{4,6}$. Immune status is also correlated with tumor formation and recurrence ${ }^{7,8}$. Many indicators, including blood neutrophil, lymphocyte, monocyte, platelet count, neutrophilocyte-to-lymphocyte ratio (NLR), derived neutrophilcyte-to-lymphocyte ratio (dNLR), lymphocyte-to-monocyte ratio (LMR) and platelet-to-lymphocyte ratio (PLR), have been reported to be prognostic predictors in various cancers ${ }^{9-13}$. A meta-analysis showed that the LMR was significantly associated with long term outcomes in colorectal cancer ${ }^{14,15}$. What is more, it has also been verified that a platelet and lymphocyte-to-monocyte ratio (COP-LMR) is a novel prognosis predictor in lung cancer ${ }^{16}$.

The prognostic nutritional index (PNI), which consists of serum albumin concentration and total lymphocyte count, is used to assess the perioperative immunonutritional status and surgical risk for patients ${ }^{5}$. It has been reported that the PNI could predict postoperative complications including the intra-abdominal abscess, postoperative cardiovascular disease and pulmonary disease, pleural effusion, ascites, urinary tract infection, intraperitoneal and subcutaneous bleeding, inflammation of the intestine, obstruction of the intestine, pancreatic fistula, lymphorrhea, and numbness of limbs in patients with colorectal cancer ${ }^{17}$. Furthermore, it is corroborated to be a prognostic factor in various tumors, including breast cancer ${ }^{17-21}$. Based on these findings, a more comprehensive scoring system, controlling nutritional status (CONUT), consisting of serum albumin, cholesterol levels and lymphocyte count in peripheral blood, is yet to be proposed to assess patient nutritional status. Similar to PNI,

${ }^{1}$ Lung Cancer Center, West China Hospital, Sichuan University, Chengdu, Sichuan, 610041, P.R. China. ${ }^{2}$ Department of Cancer, The People's Hospital of Yuechi, Guang'an, Sichuan, 638300, P.R. China. ${ }^{3}$ Department of Breast Surgery, West China Hospital of Sichuan University, Chengdu, Sichuan, 610041, P.R. China. *email: zhouqh135@163.com 


\begin{tabular}{|l|l|l|l|l|}
\hline Parameters & Normal & Light & Moderate & Severe \\
\hline Serum albumin $(\mathrm{g} / \mathrm{dL})$ & $\geq 3.50$ & $3.00-3.49$ & $2.50-2.99$ & $<2.50$ \\
\hline score & 0 & 2 & 4 & 6 \\
\hline Total lymphocyte count & $\geq 1600$ & $1200-1599$ & $800-1199$ & $<800$ \\
\hline score & 0 & 1 & 2 & 3 \\
\hline Total cholesterol $(\mathrm{mg} / \mathrm{dL})$ & $>180$ & $140-180$ & $100-139$ & $<100$ \\
\hline score & 0 & 1 & 2 & 3 \\
\hline CONUT score (total) & $0-1$ & $2-4$ & $5-8$ & $9-12$ \\
\hline Assessment & Normal & Light & Moderate & Severe \\
\hline
\end{tabular}

Table 1. The CONUT scoring system.

A Prediction for disease free survival

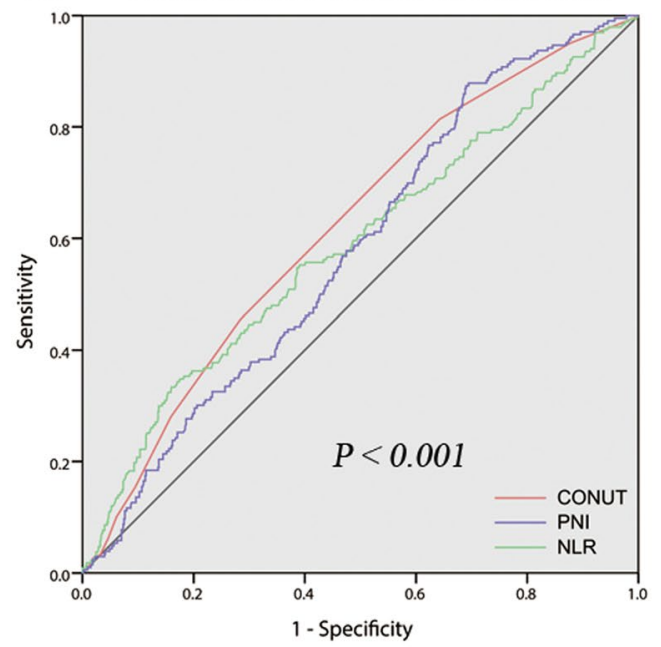

B

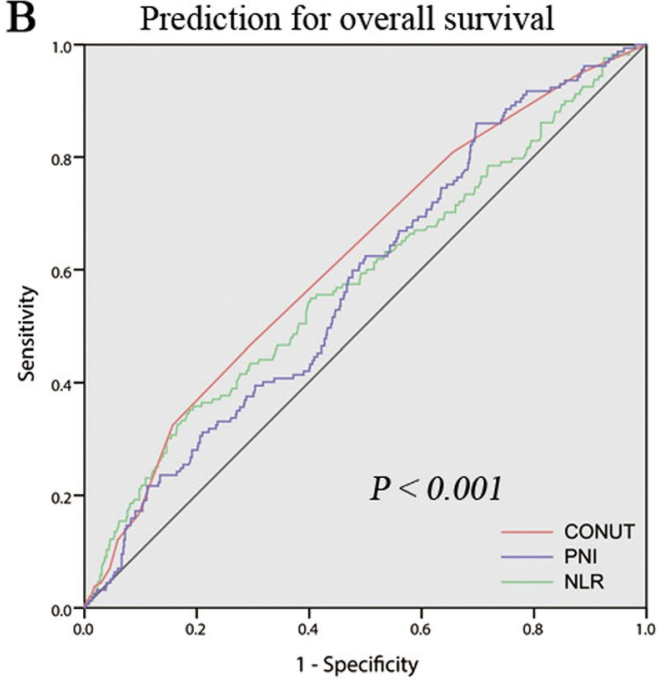

Figure 1. The ROC curves of CONUT, NLR and PNI for predicting DFS (A) and OS (B).

CONUT could easily be calculated from blood examination data. Recently, it has been shown that CONUT is an independent prognostic marker in malignant pleural mesothelioma ${ }^{22}$, resected lung squamous cell carcinoma ${ }^{23}$, gastric cancer ${ }^{4}$, and head and neck cancer ${ }^{24}$. However, its role in breast cancer has not been reported. To our knowledge, we firstly attempted to assess the prognostic significance of CONUT in breast cancer patients who received curative resection based on a large study.

\section{Materials and Methods}

Patients and follow-up. A total of 1,364 breast cancer patients who received surgical resection from 2007 to 2010 in West China Hospital of Sichuan University were recruited (Supplementary Dataset 1). The complete preoperative blood cell count was procured within seven days before surgery. The exclusion criteria were as follows: (1) patients who received chemotherapy or radiotherapy before the surgery; (2) patients with inflammatory disease or autoimmune disease; (3) patients who lacked detailed clinicopathological information; (4) male breast cancer patients. Finally, 861 cases were included in the present retrospective study. All the patients were followed up every three months in the first three years, every six months for five years, and annually within 6-10 years after the operation. Clinical check-up, laboratory examination and radiological assessment were included in the follow-up investigations.

Pathology methods and molecular subtypes. Estrogen receptor (ER), progesterone receptor (PR), human epidermal growth factor receptor 2 (HER2) statuses and Ki67 expression were assessed by immunohistochemical staining. The monoclonal ER antibody (clone SP1; Ventana, Tucson, AZ, USA), monoclonal PR (clone 1E2; Ventana), Ki-67 (clone 30-9; Ventana) and HER2 (clone 4B5; Roche, Sandhofer, Mannheim, Germany) were used. Positive ER or PR was defined as $\geq 1 \%$ of immunoreactive tumor cell nuclei, according to the American Society of Clinical Oncology and College of American Pathologists Guideline Recommendations in 2010. The cutoff value for Ki-67 was defined as $\geq 14 \%$. As for HER-2, 0 or $1+$ was negative, while $3+$ was reported as positive. Fluorescence in situ hybridization (FISH) was performed in case of a $2+$ level of staining.

The molecular subtypes were classified as Luminal A (ER+ and/or PR+, HER2-, Ki-67 < 14), Luminal B (ER+ and/or PR+, HER2 + and/or HER2-, any Ki-67), HER2-enriched (ER-, PR-, HER2+, any Ki-67), and triple-negative (ER-, PR-, HER2-, any Ki-67) breast cancer (TNBC). 


\begin{tabular}{|c|c|c|c|c|}
\hline & Total & CONUT $\leq 2$ & CONUT $\geq 3$ & $\mathbf{P}$ \\
\hline Age & & 581 & 280 & 0.003 \\
\hline$\leq 40$ & $211(24.5 \%)$ & $160(27.5 \%)$ & $51(18.2 \%)$ & \\
\hline$>40$ & $650(75.5 \%)$ & $421(72.5 \%)$ & $229(81.8 \%)$ & \\
\hline ER & & & & 0.456 \\
\hline+ & $538(62.5 \%)$ & $368(63.3 \%)$ & $170(60.7 \%)$ & \\
\hline- & $323(37.5 \%)$ & $213(36.7 \%)$ & $110(39.3 \%)$ & \\
\hline PR & & & & 0.505 \\
\hline+ & $396(46.2 \%)$ & $264(45.4 \%)$ & $134(47.9 \%)$ & \\
\hline- & $465(53.8 \%)$ & $317(54.3 \%)$ & $146(52.1 \%)$ & \\
\hline HER2 & & & & 0.253 \\
\hline+ & $198(23.0 \%)$ & $127(21.9 \%)$ & $71(25.4 \%)$ & \\
\hline- & $663(77.0 \%)$ & $454(78.1 \%)$ & $209(74.6 \%)$ & \\
\hline Ki-67 status & & & & 0.246 \\
\hline+ & $568(65.2 \%)$ & $358(63.8 \%)$ & $190(67.9 \%)$ & \\
\hline- & $293(34.8 \%)$ & $203(36.2 \%)$ & $90(32.1 \%)$ & \\
\hline pT Stage & & & & 0.003 \\
\hline 1 & $287(33.3 \%)$ & $209(37.3 \%)$ & $78(26.0 \%)$ & \\
\hline 2 & 449 (52.1\%) & $283(50.4 \%)$ & $166(55.3 \%)$ & \\
\hline 3 & $91(10.6 \%)$ & $49(8.7 \%)$ & $42(14.0 \%)$ & \\
\hline 4 & $34(3.9 \%)$ & $20(3.6 \%)$ & $14(4.7 \%)$ & \\
\hline pN Stage & & & & $\mathrm{P}<0.001$ \\
\hline 0 & $370(43.0 \%)$ & $278(47.9 \%)$ & $92(32.7 \%)$ & \\
\hline 1 & 309 (35.9\%) & $203(35.0 \%)$ & $106(37.7 \%)$ & \\
\hline 2 & $130(15.1 \%)$ & $69(11.9 \%)$ & $61(21.7 \%)$ & \\
\hline 3 & $52(6.0 \%)$ & $30(5.2 \%)$ & $22(7.8 \%)$ & \\
\hline Molecular subtype & & & & 0.095 \\
\hline Luminal A & $223(25.9 \%)$ & $162(27.9 \%)$ & $61(21.8 \%)$ & \\
\hline Luminal B & 407 (47.3\%) & $262(45.1 \%)$ & $145(51.8 \%)$ & \\
\hline HER2-enriched & $135(15.7 \%)$ & $87(15.0 \%)$ & $48(17.1 \%)$ & \\
\hline TNBC & $96(11.1 \%)$ & $70(12.0 \%)$ & $26(9.3 \%)$ & \\
\hline Histological grade & & & & 0.227 \\
\hline I-II & 585 (67.9\%) & $387(66.6 \%)$ & $198(70.7 \%)$ & \\
\hline III & $276(32.1 \%)$ & $194(33.4 \%)$ & $82(29.3 \%)$ & \\
\hline Surgery type & & & & 0.041 \\
\hline Mastectomy & 688 (79.9\%) & $453(78 \%)$ & $235(83.9 \%)$ & \\
\hline BCS & $173(20.1 \%)$ & $128(22 \%)$ & $45(16.1 \%)$ & \\
\hline Chemotherapy & & & & 0.057 \\
\hline Yes & $606(70.4 \%)$ & $397(68.3 \%)$ & $209(74.6 \%)$ & \\
\hline No & $225(29.6 \%)$ & $184(31.7 \%)$ & $71(25.4 \%)$ & \\
\hline Hormonal therapy & & & & 0.233 \\
\hline Yes & $655(76.1 \%)$ & $435(74.9 \%)$ & $220(78.6 \%)$ & \\
\hline No & 206 (26.9\%) & $146(25.1 \%)$ & $60(21.4 \%)$ & \\
\hline Radiotherapy & & & & 0.320 \\
\hline Yes & $393(45.6 \%)$ & $121(43.2 \%)$ & $272(46.8 \%)$ & \\
\hline No & 468 (54.4\%) & $159(56.8 \%)$ & $309(53.2 \%)$ & \\
\hline Target therapy & & & & 0.114 \\
\hline Yes & $125(14.5 \%)$ & $92(15.8 \%)$ & $33(11.8 \%)$ & \\
\hline No & $736(85.5 \%)$ & $489(84.2 \%)$ & $247(88.2 \%)$ & \\
\hline
\end{tabular}

Table 2. Patient and tumor characteristics by CONUT group.

Ethical approval and consent to participate. The study has been approved by the Institutional Ethical and Scientific Committee of West China Hospital of Sichuan University. Written informed consent was obtained from all participants in accordance with the policies of the committee. All methods applied within the study were performed according to the approved guidelines.

CONUT score and other scoring systems. The blood samples were investigated in one week before surgery. According to previous studies, the CONUT score was obtained based on serum albumin concentration, cholesterol level, and lymphocyte count (Table 1). The PNI was calculated by utilizing the following formula: 
A

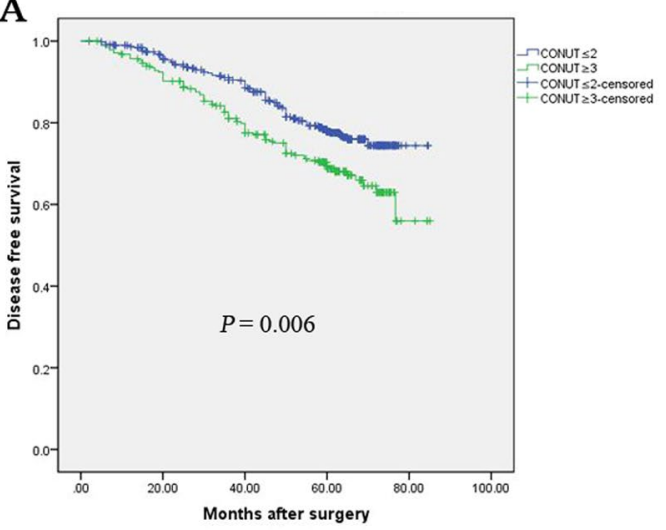

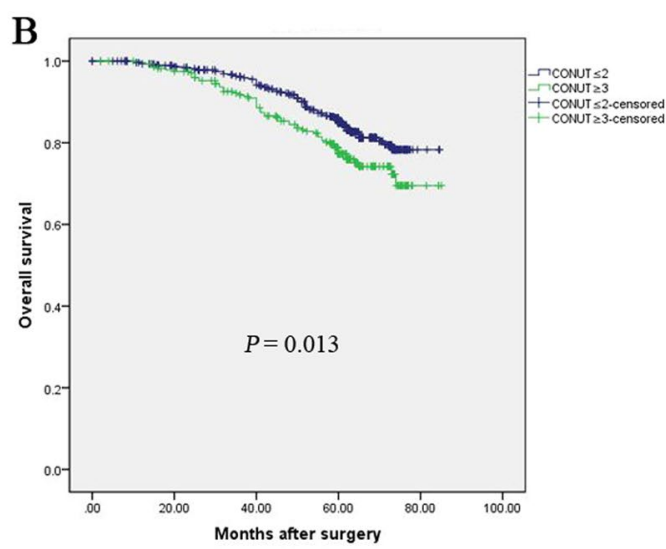

Figure 2. Kaplan-Meier survival analyses of the correlation between CONUT and survival among breast cancer patients: DFS (A) and OS (B).

$10 \times$ the serum albumin value $(\mathrm{g} / \mathrm{dl})+0.005 \times$ the total lymphocyte count in peripheral blood $\left(\right.$ per $\left.\mathrm{mm}^{3}\right)$. The neutrophil-to-lymphocyte ratio was determined as the absolute neutrophil count divided by the absolute lymphocyte count.

Determination of the cutoff value. The receiver operating characteristic (ROC) curve was used to assess the sensitivity and specificity for 5-year survival. In addition, the Youden index was calculated to choose the best cutoff value.

Statistical analysis. OS was defined as the interval from diagnoses to death of any cause or last follow-up, whichever occurred first. DFS was calculated from the time of diagnoses to the first observation of recurrence or last follow-up without evidence of recurrence. The association between clinicopathological factors and CONUT was analyzed by $X^{2}$-test. Variable was assessed on the univariate analysis, and then was calculated on the multivariable Cox proportion analysis if it was statistically significant. All statistical analyses were conducted by the SPSS (version 20.0) software pack (SPSS Inc., Chicago, IL, USA). $P<0.05$ was statistically significant.

\section{Results}

ROC analysis. Using the 5-year survival as an endpoint, 3 was considered to be the best cutoff value for CONUT since the corresponding Youden index was maximal. The sensitivity and specificity for OS were $81.6 \%$ and of $35.7 \%$, respectively (Fig. 1A,B). All the patients were classified into CONUT-low group $(\leq 2)$ and CONUThigh group $(\geq 3)$.

Comparison of CONUT with NLR or PNI. The prognostic accuracies of CONUT, PNI and NLR were explored by the AUC of the ROC curve for predicting the 5-year DFS and OS (Fig. 1A,B). The AUCs of CONUT, NLR and PNI for DFS were 0.622 (95\% CI: 0.580-0.665), 0.590 (95\% CI: 0.543-0.636), and 0.581 (95\% CI: 0.5390.624), respectively, while the AUCs of CONUT, NLR and PNI for OS were 0.621 (95\% CI: 0.573-0.669), 0.579 (95\% CI: $0.527-0.631$ ), and 0.577 (95\% CI: 0.530-0.625), respectively.

The correlation between CONUT and clinicopathological factors. Among the 861 breast cancer patients included in the present study, 223 patients were classified as luminal A subtype (25.9\%), 407 patients were Luminal B subtype (47.3\%), 135 patients were HER2 subtype (15.7\%), and 96 patients were TNBC subtype (11.1\%). The median age was 55 years old, with a median follow-up of 61.7 months. 206 patients developed tumor relapsed and 154 patients died. The clinical and pathologic characteristics of the 861 patients in the present study were presented in Table 2 . A high CONUT was significantly related with age, lymph node involvement, advanced T-stage and surgery type, but not related with Ki-67 status, high tumor grade, ER status, PR status, or HER2 over expression.

Correlations of the CONUT score with survival. The results revealed that a high CONUT was a poor prognostic factor for both DFS and OS in breast cancer patients. The 5 -year OS rates were $68.7 \%$ in the COUNT-high group and $77.9 \%$ in the COUNT-low group $(P=0.013$, Fig. $2 \mathrm{~A})$. In addition, the 5 -year DFS rates were $76.6 \%$ in the COUNT-high group and $84.6 \%$ in the COUNT-low group $(P=0.006$, Fig. $2 \mathrm{~B})$. After adjusting for p-stage, CONUT-high was still associated with worse DFS and OS in these three subgroups (Fig. 3A-F).

In the univariate analysis, high CONUT, patient age, PR status, tumor grade, T-stage, lymph node involvement and histological grade were related with DFS and OS. In the multivariate analysis, high CONUT $(P=0.07)$, patient age $(P=0.037)$, PR status $(P=0.041)$, tumor grade $(P=0.009)$, T-stage $(P=0.001)$ and lymph node involvement post-surgery $(P=0.002)$ were independent predictors of DFS, while high CONUT $(P=0.027)$, patient age $(P=0.042)$, tumor grade $(P=0.003)$, T-stage $(P=0.031)$ and lymph nodes $(P=0.002)$ were correlated with OS (Tables 3 and 4 ). 
A

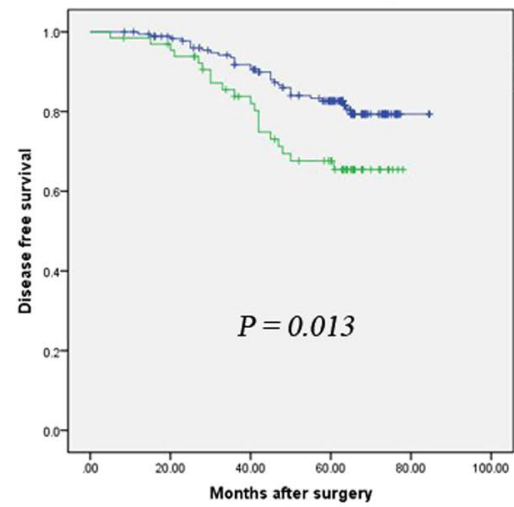

C

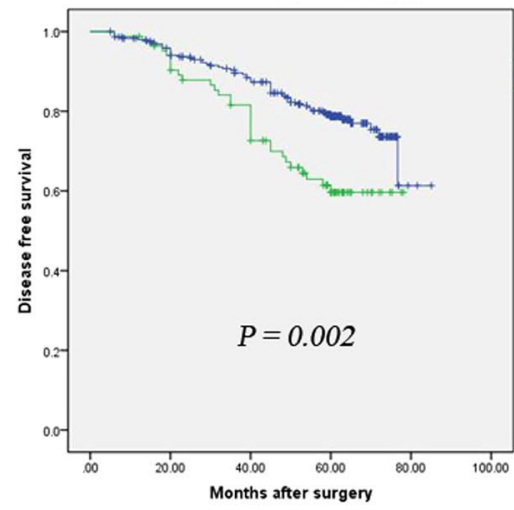

E

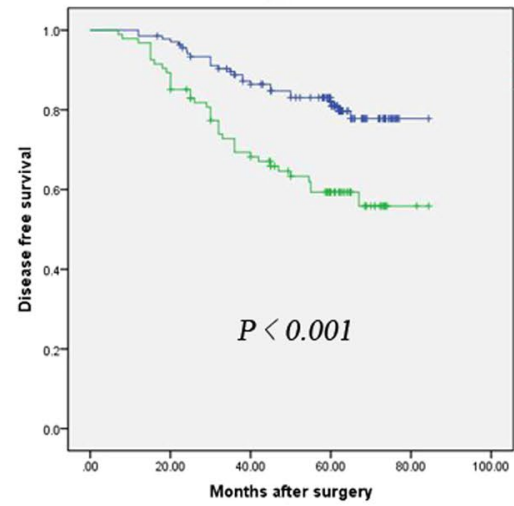

Stage I

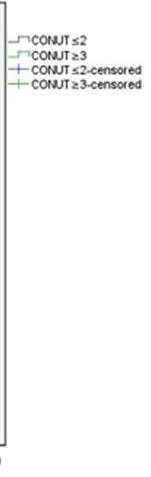

Stage II

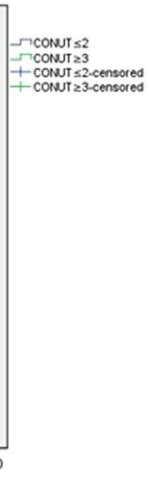

Stage III

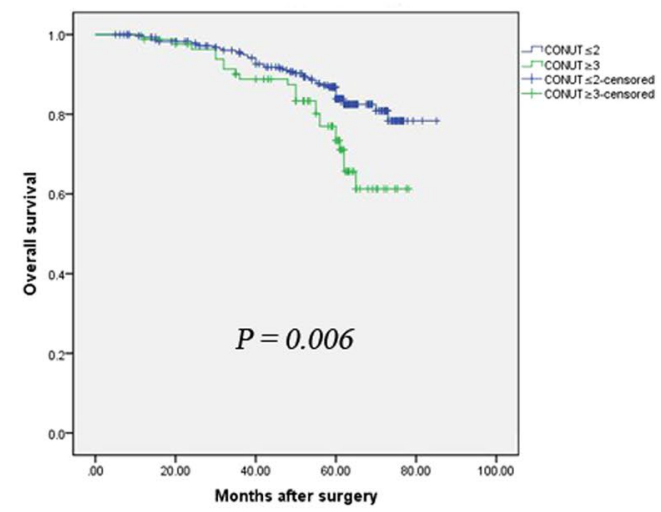

F

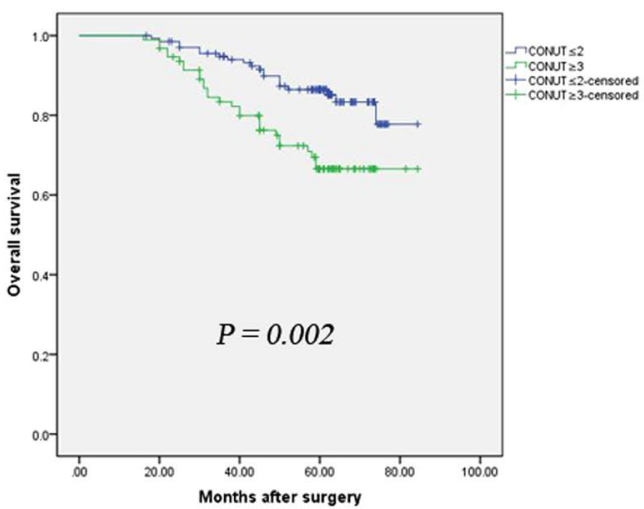

Figure 3. Kaplan-Meier survival analyses of DFS and OS, according to CONUT, among patients in the stage I, stage II and stage III subgroups.

Since there are four molecular subtypes for breast cancer, the prognostic value of CONUT was subsequently analyzed in these four subgroups. The results revealed obvious associations of high CONUT score and worse outcomes in the luminal B subgroup (Tables 5 and 6).

\section{Discussion}

Studies have recently demonstrated the impact of CONUT on prognosis in several malignancies. In the present study, the prognostic value of CONUT in female breast cancer was initially assessed. Our results showed that CONUT was more accurate in prognosis prediction, when compared with previously reported prognostic scoring systems, PNI or NLR. Furthermore, the results indicated that CONUT was associated with age, tumor size and invasion. Importantly, CONUT independently predicted the prognosis of breast cancer patients, regardless of the tumor stage. Patients with high CONUT predicted the shorter DFS and OS, when compared with patients with low CONUT. Overall, these results suggested that CONUT might be a prognostic factor in breast cancer 


\begin{tabular}{|c|c|c|c|c|}
\hline & Univariate analysis & & Multivariate analysi & \\
\hline & HR (95\% CI) & P value & HR $(95 \% \mathrm{CI})$ & Pvalue \\
\hline CONUT & $1.486(1.118-1.975)$ & 0.006 & $1.548(1.127-2.125)$ & 0.07 \\
\hline $\mathrm{CONUT} \leq 2$ & & & & \\
\hline $\mathrm{CONUT} \geq 3$ & & & & \\
\hline Patient age & $0.673(0.497-0.910)$ & 0.01 & $0.705(0.507-0.980)$ & 0.037 \\
\hline$\leq 40$ & & & & \\
\hline$>40$ & & & & \\
\hline ER & $1.127(0.812-1.564)$ & 0.475 & & \\
\hline+ & & & & \\
\hline- & & & & \\
\hline PR & $0.715(0.536-0.954)$ & 0.022 & $0.737(0.550-0.988)$ & 0.041 \\
\hline- & & & & \\
\hline+ & & & & \\
\hline HER2 & $0.874(0.630-1.211)$ & 0.418 & & \\
\hline- & & & & \\
\hline+ & & & & \\
\hline Ki-67 status & $1.276(0.930-1.749)$ & 0.131 & & \\
\hline- & & & & \\
\hline+ & & & & \\
\hline pT Stage & $1.404(1.234-1.597)$ & $<0.001$ & $1.307(1.123-1.522)$ & 0.001 \\
\hline 1 & & & & \\
\hline 2 & & & & \\
\hline 3 & & & & \\
\hline 4 & & & & \\
\hline pN Stage & $1.518(1.307-1.764)$ & $<0.001$ & $1.333(1.098-1.599)$ & 0.002 \\
\hline 0 & & & & \\
\hline 1 & & & & \\
\hline 2 & & & & \\
\hline 3 & & & & \\
\hline Molecular subtype & $0.983(0.846-1.142)$ & 0.824 & & \\
\hline Luminal A & & & & \\
\hline Luminal B & & & & \\
\hline HER2-enriched & & & & \\
\hline TNBC & & & & \\
\hline Histological grade & $1.587(1.723-2.15)$ & $<0.001$ & $1.476(1.101-1.979)$ & 0.009 \\
\hline I-II & & & & \\
\hline III & & & & \\
\hline Surgery type & $1.112(0.823-1.486)$ & 0.456 & & \\
\hline Mastectomy & & & & \\
\hline BCS & & & & \\
\hline Chemotherapy & $0.931(0.756-1.268)$ & 0.631 & & \\
\hline No & & & & \\
\hline Yes & & & & \\
\hline Hormone therapy & $0.867(0.754-1.625)$ & 0.374 & & \\
\hline No & & & & \\
\hline Yes & & & & \\
\hline Radiotherapy & $1.09(0.826-1.468)$ & 0.561 & & \\
\hline No & & & & \\
\hline Yes & & & & \\
\hline Target therapy & $1.159(0.876-1.542)$ & 0.32 & & \\
\hline No & & & & \\
\hline Yes & & & & \\
\hline
\end{tabular}

Table 3. Analyses regarding the prognostic factors for disease free survival.

patients undergoing potentially curative resection. To our knowledge, our study firstly demonstrated the association between preoperative CONUT and clinicopathological factors or survival in breast cancer patients who underwent resection. 


\begin{tabular}{|c|c|c|c|c|}
\hline & \multicolumn{2}{|l|}{ Univariate analysis } & \multicolumn{2}{|l|}{ Multivariate analysis } \\
\hline & HR $(95 \% \mathrm{CI})$ & P value & HR $(95 \% \mathrm{CI})$ & P value \\
\hline CONUT & $1.514(1.108-2.198)$ & 0.013 & $1.220(1.023-1.455)$ & 0.027 \\
\hline \multicolumn{5}{|l|}{ CONUT $\leq 2$} \\
\hline \multicolumn{5}{|l|}{ CONUT $\geq 3$} \\
\hline Patient age & $0.669(0.472-0.947)$ & 0.023 & $0.673(0.460-0.985)$ & 0.042 \\
\hline \multicolumn{5}{|l|}{$\leq 40$} \\
\hline \multicolumn{5}{|l|}{$>40$} \\
\hline ER & $1.346(0.906-1.999)$ & 0.141 & & \\
\hline \multicolumn{5}{|l|}{-} \\
\hline \multicolumn{5}{|l|}{+} \\
\hline PR & $0.684(0.490-0.956)$ & 0.026 & $0.721(0.502-1.034)$ & 0.076 \\
\hline \multicolumn{5}{|l|}{-} \\
\hline \multicolumn{5}{|l|}{+} \\
\hline HER2 & $0.879(0.605-1.279)$ & 0.501 & & \\
\hline \multicolumn{5}{|l|}{+} \\
\hline \multicolumn{5}{|l|}{-} \\
\hline Ki-67 status & $1.161(0.811-1.662)$ & 0.415 & & \\
\hline \multicolumn{5}{|l|}{+} \\
\hline \multicolumn{5}{|l|}{-} \\
\hline pT Stage & $1.452(1.257-1.678)$ & $<0.001$ & $1.219(1.017-1.462)$ & 0.031 \\
\hline \multicolumn{5}{|l|}{1} \\
\hline \multicolumn{5}{|l|}{2} \\
\hline \multicolumn{5}{|l|}{3} \\
\hline \multicolumn{5}{|l|}{4} \\
\hline pN Stage & $1.582(1.338-1.870)$ & $<0.001$ & $1.401(1.135-1.730)$ & 0.002 \\
\hline \multicolumn{5}{|l|}{0} \\
\hline \multicolumn{5}{|l|}{1} \\
\hline 2 & & & & \\
\hline 3 & & & & \\
\hline Molecular subtype & $0.901(0.854-1.076)$ & 0.250 & & \\
\hline Luminal A & & & & \\
\hline Luminal B & & & & \\
\hline HER2-enriched & & & & \\
\hline TNBC & & & & \\
\hline Histological grade & $1.683(1.274-1.792)$ & $<0.001$ & $1.635(1.193-2.381)$ & 0.003 \\
\hline I-II & & & & \\
\hline III & & & & \\
\hline Surgery type & $1.077(0.821-1.46)$ & 0.62 & & \\
\hline Mastectomy & & & & \\
\hline BCS & & & & \\
\hline Chemotherapy & $0.932(0.721-1.236)$ & 0.718 & & \\
\hline Yes & & & & \\
\hline No & & & & \\
\hline Hormone therapy & $0.905(0.678-1.205)$ & 0.462 & & \\
\hline Yes & & & & \\
\hline No & & & & \\
\hline Radiotherapy & $1.036(0.774-1.387)$ & 0.812 & & \\
\hline Yes & & & & \\
\hline No & & & & \\
\hline Target therapy & $1.119(0.836-1.498)$ & 0.45 & & \\
\hline Yes & & & & \\
\hline No & & & & \\
\hline
\end{tabular}

Table 4. Analyses regarding the prognostic factors for overall survival.

CONUT was originally reported as an efficient tool for the early detection and continuous control of hospital undernutrition ${ }^{25}$. Forward studies have demonstrated that CONUT has a prognostic impact on patients with severely decompensated acute heart failure ${ }^{26,27}$. Since CONUT was based on the serum albumin level, total 


\begin{tabular}{|l|l|l|l|l|}
\hline \multirow{4}{*}{} & \multicolumn{3}{|l|}{ Univariate analysis } & \multicolumn{2}{l|}{ Multivariate analysis } \\
\cline { 2 - 5 } & HR $(\mathbf{9 5} \% \mathbf{C I})$ & $\mathbf{p}$ value & HR $(\mathbf{9 5} \% \mathbf{C I})$ & p value \\
\hline Luminal A & $1.516(0.781-2.945)$ & 0.219 & & \\
\hline CONUT $\leq 2$ & & & & \\
\hline CONUT $\geq 3$ & & & & \\
\hline Luminal B & $1.704(1.153-2.519)$ & 0.007 & $1.604(1.065-2.414)$ & 0.024 \\
\hline CONUT $\leq 2$ & & & & \\
\hline CONUT $\geq 3$ & & & & \\
\hline TNBC & $2.272(1.091-4.731)$ & 0.028 & $1.423(0.917-2.209)$ & 0.116 \\
\hline CONUT $\leq 2$ & & & & \\
\hline CONUT $\geq 3$ & & & & \\
\hline HER2-enriched & $2.398(1.076-5.346)$ & 0.032 & $1.925(0.715-5.180)$ & 0.195 \\
\hline CONUT $\leq 2$ & & & & \\
\hline CONUT $\geq 3$ & & & & \\
\hline
\end{tabular}

Table 5. Analyses results of CONUT for the prediction of disease free survival in different breast cancer subtypes.

\begin{tabular}{|l|l|l|l|l|}
\hline \multirow{2}{*}{} & \multicolumn{3}{|l|}{ Univariate analysis } & \multicolumn{2}{l|}{ Multivariate analysis } \\
\cline { 2 - 5 } & HR $(\mathbf{9 5} \% \mathbf{C I})$ & $\mathbf{p ~ v a l u e}$ & HR $(\mathbf{9 5} \% \mathbf{C I})$ & $\mathbf{p}$ value \\
\hline Luminal A & $1.403(0.661-2.980)$ & 0.378 & & \\
\hline CONUT $\leq 2$ & & & & \\
\hline CONUT $\geq 3$ & & & & \\
\hline Luminal B & $2.213(1.388-3.530)$ & 0.001 & $1.878(1.154-3.055)$ & 0.01 \\
\hline CONUT $\leq 2$ & & & & \\
\hline CONUT $\geq 3$ & & & & \\
\hline TNBC & $1.429(0.662-3.081)$ & 0.363 & & \\
\hline CONUT $\leq 2$ & & & & \\
\hline CONUT $\geq 3$ & & & & \\
\hline HER2-enriched & $2.542(1.067-6.465)$ & 0.056 & & \\
\hline CONUT $\leq 2$ & & & & \\
\hline CONUT $\geq 3$ & & & & \\
\hline
\end{tabular}

Table 6. Analyses results of CONUT for the prediction of overall survival in different breast cancer subtypes.

cholesterol level and total lymphocyte count, the CONUT score could reflect the malnutrition and systemic inflammation status. Besides, tumor progression and treatment tolerance have been revealed to be closely correlated with the nutritional and inflammation status. Thus, CONUT could theoretically be a comprehensive prognostic factor. In the present study, the results show that CONUT is associated with both DFS and OS for all the included patients, and a high CONUT score might be associated with a poor prognosis.

PNI and NLR are both reported scoring systems for the evaluation of the general condition of patients and have been demonstrated to be related with cancer survival, including breast cancer ${ }^{2,28,29}$. Comparisons between CONUT and PNI, previous results suggested that CONUT tended to be more superior to the PNI scoring systems for the prediction of survival in various cancer patients. In the present study, our results suggested that CONUT was proved to be superior to both PNI and LNR for the prognosis prediction in resected breast cancer patients.

Among the three components of CONUT, serum albumin concentration is the most important parameter, which is twice the weight of the other two. It is a reliable indicator not only for nutritional status but also for systemic inflammation ${ }^{30,31}$. Studies demonstrated that low serum albumin was associated with poor survival and increased risk of cancer-related death in breast cancer patients ${ }^{32,33}$. Besides, pro-inflammatory cytokines (such as IL- 6 or TNF- $\alpha$ ) and CRP could also cause decreased serum albumin concentration and modulate albumin synthesis via hepatocytes ${ }^{34-36}$. As cholesterol plays a crucial role in forming cell membranes, cholesterol is related with numerous biochemical pathways which are potentially correlated immune response besides tumorigenesis ${ }^{37-39}$. It has also been reported that low cholesterol level is correlated with poor prognosis in various malignancies as the cholesterol may affect the caloric intake and cell membrane formation ${ }^{40,41}$. What is more, low peripheral lymphocyte count is an indicator for the inadequate host immune response and is correlated to undesirable prognosis in various cancers, including breast cancer ${ }^{9,42,43}$. Thus, the combination of these three parameters could integrate the accuracy of each parameter to assess for the general condition.

\section{Conclusion}

The present study indicates that CONUT is a useful prognostic factor for breast cancer patients undergoing curative resection, and a high CONUT score might be associated with a poor prognosis. 


\section{Data availability}

All data generated or analyzed during this study are included in this published article.

Received: 23 November 2018; Accepted: 27 March 2020;

Published online: 20 April 2020

\section{References}

1. Torre, L. A. et al. Global cancer statistics, 2012. CA: a cancer journal for clinicians $\mathbf{6 5}, 87-108$, https://doi.org/10.3322/caac.21262 (2015).

2. Chen, J. et al. Meta-analysis of prognostic value of inflammation parameter in breast cancer. Journal of cancer research and therapeutics 14, S85-S89, https://doi.org/10.4103/0973-1482.160917 (2018).

3. Elinav, E. et al. Inflammation-induced cancer: crosstalk between tumours, immune cells and microorganisms. Nature reviews. Cancer 13, 759-771, https://doi.org/10.1038/nrc3611 (2013).

4. Kuroda, D. et al. Controlling Nutritional Status (CONUT) score is a prognostic marker for gastric cancer patients after curative resection. Gastric cancer: official journal of the International Gastric Cancer Association and the Japanese Gastric Cancer Association 21, 204-212, https://doi.org/10.1007/s10120-017-0744-3 (2018).

5. Migita, K. et al. The prognostic nutritional index predicts long-term outcomes of gastric cancer patients independent of tumor stage. Annals of surgical oncology 20, 2647-2654, https://doi.org/10.1245/s10434-013-2926-5 (2013).

6. Sakurai, K. et al. Predictive Potential of Preoperative Nutritional Status in Long-Term Outcome Projections for Patients with Gastric Cancer. Annals of surgical oncology 23, 525-533, https://doi.org/10.1245/s10434-015-4814-7 (2016).

7. Huang, Y. et al. Impacts of Preoperative Serum Albumin Level on Outcomes of Cytoreductive Surgery and Perioperative Intraperitoneal Chemotherapy. Annals of surgical oncology 23, 2411-2418, https://doi.org/10.1245/s10434-016-5172-9 (2016).

8. Prado, C. M. et al. Prevalence and clinical implications of sarcopenic obesity in patients with solid tumours of the respiratory and gastrointestinal tracts: a population-based study. The Lancet. Oncology 9, 629-635, https://doi.org/10.1016/s1470-2045(08)70153-0 (2008).

9. Karantanos, T., Karanika, S., Seth, B. \& Gignac, G. The absolute lymphocyte count can predict the overall survival of patients with non-small cell lung cancer on nivolumab: a clinical study. Clinical \& translational oncology: official publication of the Federation of Spanish Oncology Societies and of the National Cancer Institute of Mexico, https://doi.org/10.1007/s12094-018-1908-2 (2018).

10. Lee, J. S., Kim, N. Y., Na, S. H., Youn, Y. H. \& Shin, C. S. Reference values of neutrophil-lymphocyte ratio, lymphocyte-monocyte ratio, platelet-lymphocyte ratio, and mean platelet volume in healthy adults in South Korea. Medicine 97, e11138, https://doi. org $/ 10.1097 / \mathrm{md} .0000000000011138$ (2018).

11. Mellor, K. L., Powell, A. \& Lewis, W. G. Systematic Review and Meta-Analysis of the Prognostic Significance of NeutrophilLymphocyte Ratio (NLR) After R0 Gastrectomy for Cancer. Journal of gastrointestinal cancer, https://doi.org/10.1007/s12029-0180127-y (2018).

12. Mizuguchi, S., Izumi, N., Tsukioka, T., Komatsu, H. \& Nishiyama, N. Neutrophil-lymphocyte ratio predicts recurrence in patients with resected stage 1 non-small cell lung cancer. Journal of cardiothoracic surgery 13, 78, https://doi.org/10.1186/s13019-018-0763-0 (2018).

13. Zhang, L. X., Wei, Z. J. \& Xu, A. M. Can the neutrophil-lymphocyte ratio and platelet-lymphocyte ratio be beneficial in predicting lymph node metastasis and promising prognostic markers of gastric cancer patients? Tumor maker retrospective study. International journal of surgery (London, England), https://doi.org/10.1016/j.ijsu.2018.06.037 (2018).

14. Tan, D., Fu, Y., Tong, W. \& Li, F. Prognostic significance of lymphocyte to monocyte ratio in colorectal cancer: A meta-analysis. International journal of surgery (London, England) 55, 128-138, https://doi.org/10.1016/j.ijsu.2018.05.030 (2018).

15. Rosin, R. D. International Journal of Surgery. Editor's perspectives-June 2015. International journal of surgery (London, England) 18, 245-246, https://doi.org/10.1016/j.ijsu.2015.05.031 (2015).

16. Lim, J. U. et al. Prognostic value of platelet count and lymphocyte to monocyte ratio combination in stage IV non-small cell lung cancer with malignant pleural effusion. PloS one 13, e0200341, https://doi.org/10.1371/journal.pone.0200341 (2018).

17. Tokunaga, R. et al. Prognostic Nutritional Index Predicts Severe Complications, Recurrence, and Poor Prognosis in Patients With Colorectal Cancer Undergoing Primary Tumor Resection. Diseases of the colon and rectum 58, 1048-1057, https://doi.org/10.1097/ dcr.0000000000000458 (2015).

18. Mori, S. et al. The Significance of the Prognostic Nutritional Index in Patients with Completely Resected Non-Small Cell Lung Cancer. PloS one 10, e0136897, https://doi.org/10.1371/journal.pone.0136897 (2015).

19. Qiu, C. et al. Evaluation of Prognostic Nutritional Index in Patients Undergoing Radical Surgery with Nonsmall Cell Lung Cancer. Nutrition and cancer 67, 741-747, https://doi.org/10.1080/01635581.2015.1032430 (2015).

20. Sun, K., Chen, S., Xu, J., Li, G. \& He, Y. The prognostic significance of the prognostic nutritional index in cancer: a systematic review and meta-analysis. Journal of cancer research and clinical oncology 140, 1537-1549, https://doi.org/10.1007/s00432-014-1714-3 (2014).

21. Yao, Z. H. et al. Prognostic nutritional index predicts outcomes of malignant pleural mesothelioma. Journal of cancer research and clinical oncology 139, 2117-2123, https://doi.org/10.1007/s00432-013-1523-0 (2013).

22. Takamori, S. et al. The Controlling Nutritional Status Score Is a Significant Independent Predictor of Poor Prognosis in Patients With Malignant Pleural Mesothelioma. Clinical lung cancer 18, e303-e313, https://doi.org/10.1016/j.cllc.2017.01.008 (2017).

23. Toyokawa, G. et al. Prognostic impact of controlling nutritional status score in resected lung squamous cell carcinoma. Journal of thoracic disease 9, 2942-2951, https://doi.org/10.21037/jtd.2017.07.108 (2017).

24. Kono, T., Sakamoto, K., Shinden, S. \& Ogawa, K. Pre-therapeutic nutritional assessment for predicting severe adverse events in patients with head and neck cancer treated by radiotherapy. Clinical nutrition (Edinburgh, Scotland) 36, 1681-1685, https://doi. org/10.1016/j.clnu.2016.10.021 (2017).

25. Ignacio de Ulibarri, J. et al. CONUT: a tool for controlling nutritional status. First validation in a hospital population. Nutricion hospitalaria 20, 38-45 (2005).

26. Shirakabe, A. et al. The prognostic impact of malnutrition in patients with severely decompensated acute heart failure, as assessed using the Prognostic Nutritional Index (PNI) and Controlling Nutritional Status (CONUT) score. Heart and vessels 33, 134-144, https://doi.org/10.1007/s00380-017-1034-z (2018).

27. Lopez-Larramona, G., Lucendo, A. J. \& Tenias, J. M. Association between nutritional screening via the Controlling Nutritional Status index and bone mineral density in chronic liver disease of various etiologies. Hepatology research: the official journal of the Japan Society of Hepatology 45, 618-628, https://doi.org/10.1111/hepr.12395 (2015).

28. Qiu, X., Song, Y., Cui, Y. \& Liu, Y. Increased neutrophil-lymphocyte ratio independently predicts poor survival in non-metastatic triple-negative breast cancer patients. IUBMB life 70, 529-535, https://doi.org/10.1002/iub.1745 (2018).

29. Zhu, M. et al. Pretreatment neutrophil-lymphocyte and platelet-lymphocyte ratio predict clinical outcome and prognosis for cervical Cancer. Clinica chimica acta; international journal of clinical chemistry 483, 296-302, https://doi.org/10.1016/j. cca.2018.05.025 (2018). 
30. Cengiz, O., Kocer, B., Surmeli, S., Santicky, M. J. \& Soran, A. Are pretreatment serum albumin and cholesterol levels prognostic tools in patients with colorectal carcinoma? Medical science monitor: international medical journal of experimental and clinical research. 12, CR240-247 (2006).

31. de Ulibarri Perez, J. I., Fernandez, G., Rodriguez Salvanes, F. \& Diaz Lopez, A. M. Nutritional screening; control of clinical undernutrition with analytical parameters. Nutricion hospitalaria 29, 797-811, https://doi.org/10.3305/nh.2014.29.4.7275 (2014).

32. Lis, C. G., Grutsch, J. F., Vashi, P. G. \& Lammersfeld, C. A. Is serum albumin an independent predictor of survival in patients with breast cancer? JPEN. Journal of parenteral and enteral nutrition 27, 10-15, https://doi.org/10.1177/014860710302700110 (2003).

33. Cui, L. et al. Pituitary tumor transforming gene: a novel therapeutic target for glioma treatment. Acta Biochim Biophys Sin (Shanghai) 47, 414-421, https://doi.org/10.1093/abbs/gmv026 (2015).

34. Mohri, Y. et al. Prognostic nutritional index predicts postoperative outcome in colorectal cancer. World journal of surgery 37, 2688-2692, https://doi.org/10.1007/s00268-013-2156-9 (2013).

35. Peters, S. J. et al. Co-culture of primary rat hepatocytes with rat liver epithelial cells enhances interleukin-6-induced acute-phase protein response. Cell and tissue research 340, 451-457, https://doi.org/10.1007/s00441-010-0955-y (2010).

36. Honda, H. et al. Serum albumin, C-reactive protein, interleukin 6, and fetuin a as predictors of malnutrition, cardiovascular disease, and mortality in patients with ESRD. American journal of kidney diseases: the official journal of the National Kidney Foundation 47, 139-148, https://doi.org/10.1053/j.ajkd.2005.09.014 (2006).

37. Strasak, A. M. et al. Time-dependent association of total serum cholesterol and cancer incidence in a cohort of 172,210 men and women: a prospective 19-year follow-up study. Annals of oncology: official journal of the European Society for Medical Oncology 20, 1113-1120, https://doi.org/10.1093/annonc/mdn736 (2009).

38. Ko, K. et al. Influence of nutritional deficiency on prognosis of renal cell carcinoma (RCC). BJU international 112, 775-780, https:// doi.org/10.1111/bju.12275 (2013)

39. Iribarren, C., Reed, D. M., Chen, R., Yano, K. \& Dwyer, J. H. Low serum cholesterol and mortality. Which is the cause and which is the effect? Circulation 92, 2396-2403 (1995).

40. Kang, R. et al. Apolipoprotein E epsilon 2 allele and low serum cholesterol as risk factors for gastric cancer in a Chinese Han population. Scientific reports 6, 19930, https://doi.org/10.1038/srep19930 (2016).

41. Niendorf, A., Nagele, H., Gerding, D., Meyer-Pannwitt, U. \& Gebhardt, A. Increased LDL receptor mRNA expression in colon cancer is correlated with a rise in plasma cholesterol levels after curative surgery. International journal of cancer 61, 461-464 (1995).

42. Vicente Conesa, M. A. et al. Predictive value of peripheral blood lymphocyte count in breast cancer patients treated with primary chemotherapy. Breast (Edinburgh, Scotland) 21, 468-474, https://doi.org/10.1016/j.breast.2011.11.002 (2012).

43. Hoskin, P. J., Rojas, A. M., Peiris, S. N., Mullassery, V. \& Chong, I. Y. Pre-treatment haemoglobin and peripheral blood lymphocyte count as independent predictors of outcome in carcinoma of cervix. Clinical oncology (Royal College of Radiologists (Great Britain)) 26, 179-184, https://doi.org/10.1016/j.clon.2013.11.023 (2014).

\section{Acknowledgements}

We thank all practitioners who participated in the documentation of the data. This study was supported by the Grants from Science and Technology Bureau of Sichuan province (No. 2017RZ0045).

\section{Author contributions}

Q.H.Z. conceived the study. W.L., M.L., T.W., Y.F.D., G.Z.M. and Z.K.L., were responsible for data curation and approval of the manuscript. G.Z.M., Q.W., X.J.L. and D.P. performed the analyses and prepared figure and tables. All authors reviewed the manuscript.

\section{Competing interests}

The authors declare no competing interests.

\section{Additional information}

Supplementary information is available for this paper at https://doi.org/10.1038/s41598-020-63610-7.

Correspondence and requests for materials should be addressed to Q.Z.

Reprints and permissions information is available at www.nature.com/reprints.

Publisher's note Springer Nature remains neutral with regard to jurisdictional claims in published maps and institutional affiliations.

(c) (i) Open Access This article is licensed under a Creative Commons Attribution 4.0 International c. License, which permits use, sharing, adaptation, distribution and reproduction in any medium or format, as long as you give appropriate credit to the original author(s) and the source, provide a link to the Creative Commons license, and indicate if changes were made. The images or other third party material in this article are included in the article's Creative Commons license, unless indicated otherwise in a credit line to the material. If material is not included in the article's Creative Commons license and your intended use is not permitted by statutory regulation or exceeds the permitted use, you will need to obtain permission directly from the copyright holder. To view a copy of this license, visit http://creativecommons.org/licenses/by/4.0/.

(C) The Author(s) 2020 